Proceedings of XIX International Scientific Conference "New Technologies and Achievements in Metallurgy, Material Engineering, Production Engineering and Physics", Częstochowa, Poland, June 7-8, 2018

\title{
Analysis of Thermal Radiation in the Heating of Steel Round Bar Bundles
}

\author{
R. WYCZÓEKOWSKI* AND J. BORYCA \\ Department of Industrial Furnaces and Environmental Protection, \\ Faculty of Production Engineering and Materials Technology, Czestochowa University of Technology, \\ al. Armii Krajowej 19, 42-200 Czestochowa, Poland
}

\begin{abstract}
The article analyses the share of thermal radiation $Q_{r d}$ in the process of heating up the bundles of steel round bars during the heat treatment. For this purpose the own calculation model based on the analogy of thermoelectricity was used. In proposed approach to each heat transfer mechanism the appropriate thermal resistance is assigned. Then, based on the geometric model of the considered medium in the form of so-called elementary cell, the effective thermal conductivity $k_{e f}$ was calculated. Coefficient $k_{e f}$ was calculated for two cases, first considering all heat transfer mechanisms and then omitting thermal radiation. Because coefficient calculated for the second case concerns only thermal conduction phenomenon, it was called substantial thermal conductivity and was marked as $k_{s u}$. Share of thermal radiation in the total heat transfer $Q_{r d}$ was calculated by comparison the values of $k_{e f}$ and $k_{s u}$ coefficients.
\end{abstract}

DOI: 10.12693/APhysPolA.135.256

PACS/topics: heat treatment, steel bars, bar bundle, thermal radiation, effective thermal conductivity

\section{Introduction}

Steel round bars belong to the basic products of the steel industry. They are widely used in various areas of the economy as finished or semifinished products. In many cases, in order to give the bars the required mechanical and technological properties, these elements are heat treated. During these operations, the bars are most often heated in the form of cylindrically shaped bundles $[1,2]$. An example of bar bundles heated in a soaking furnace is shown in Fig. 1. Bar bundle is a very specific type of charge, because due to its internal structure it is a porous medium. Between the individual bars there occur spaces filled with gas, which is the medium filling the heating chamber of the furnace.

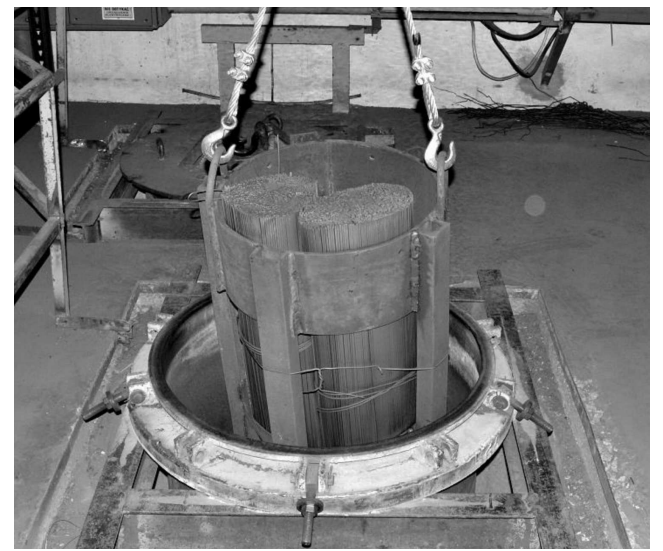

Fig. 1. Bar bundles heat treated in a soaking furnace.

*corresponding author; e-mail: rwyczolkowski@wip.pcz.pl

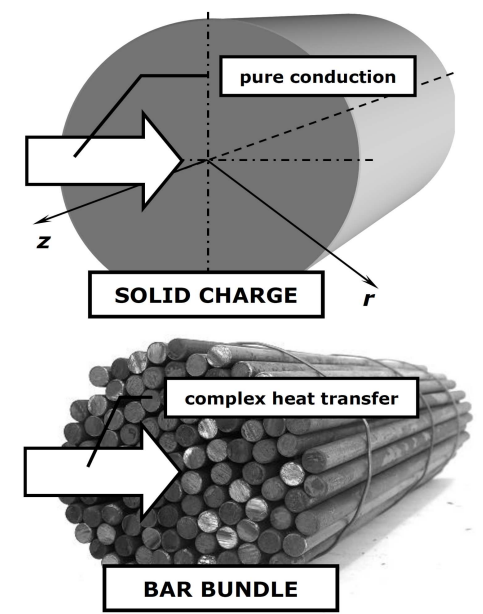

Fig. 2. Different character of heat transfer phenomenon in a solid charge and in a round bar bundle.

Due to the dimensional diversity of the bar bundles, the heat in its area flow mainly in the radial direction. In this direction, the bundles are characterized by a granular structure. This feature makes that the heat transfer in the bundle area has complex character. This process is a combination of the following mechanisms: conduction across individual bars, conduction in free spaces filled by gas, contact conduction between adjacent bars and thermal radiation between surfaces of bars. For this reason, the process of bundle heating is substantially different than the thermal conduction occurring in the case of heating a solid charge. The differentness of these phenomena is schematically shown in Fig. 2.

The control of the heat treatment process requires accurate knowledge about the current amount of heat transferred within the charge $[3,4]$. This problem has 
crucial meaning because heat treatment operations significantly bear on such performance metrics of the plant as: productivity, energy consumption, product quality, and emission of pollutants [5]. Determination of the amount of heat transferred within the bar bundle requires knowledge about the shares of individual heat transfer mechanisms. What is important for this phenomenon, the contribution of each mechanism in total heat transfer varies with charge temperature. The article presents the calculation methodology on which the percentage share of thermal radiation at the heating of round bars bundle was determined.

\section{Analysis and modelling}

The process of heat transfer within a bar bundle in a quantitative way can be expressed by the effective thermal conductivity $k_{e f}$. This parameter is commonly used in the theory of porous media $[6,7]$. The effective thermal conductivity of the considered charge can be calculated by applying the model based on the analysis of thermal resistances of individual modes of heat transfer $[8,9]$. In this case there are taken: conduction resistance in bars $R_{b r}$, conduction resistance in gas $R_{g s}$, thermal contact resistance $R_{c t}$ and radiation resistance $R_{r d}$.
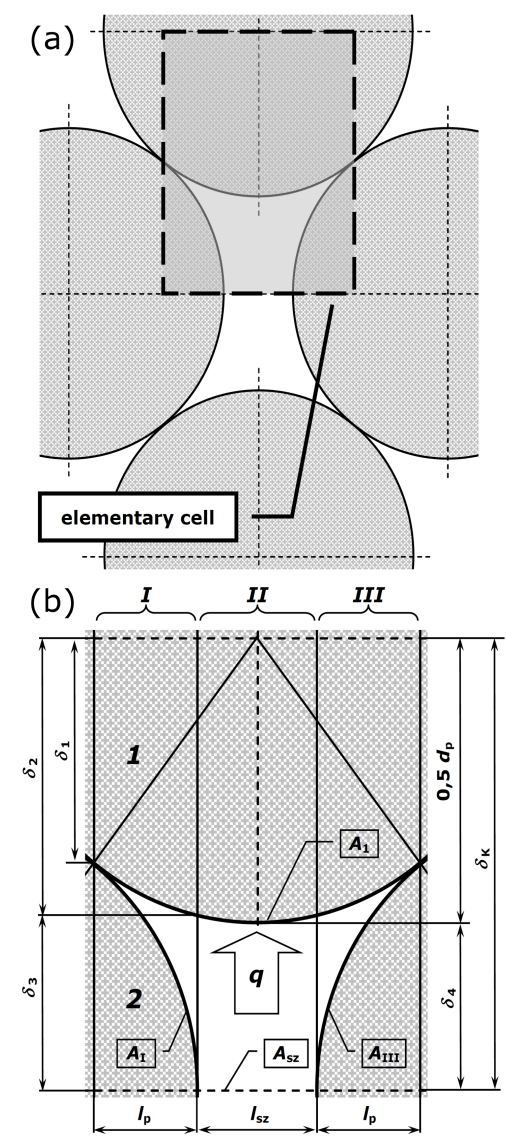

Fig. 3. (a) Repeatable fragment of considered charge, (b) elementary cell used to analysis of thermal resistances.
The physical model of the analyzed medium is the starting point for further analysis. This is a bed of round bars with a staggered arrangement. This means that the bars from successive layers are shifted in relation to each other. The result of this arrangement are free spaces enclosed by the surfaces of four bars located in three layers. A repeatable fragment of described bed is shown in Fig. 3a. The basis for the derivation of all mathematical relations is isolated from this fragment the elementary cell of heat transfer (Fig. 3b). This cell is divided into three vertical sections, a central section (II) and two identical side sections (I, III). These sections are parallel to the vector of heat flux $q$. The symbols $A_{1}$, $A_{I}, A_{1 I I}$ and $A_{s z}$ indicate the areas of surfaces involved in the radiation heat transfer. To solve the problem, in addition to the geometrical conditions, the heat flow conditions should also be given. It is assumed that the bars from one layer have the same temperature, while the temperature difference occurs between successive layers. The effect of such a temperature field is a onedimensional, vertical heat flow. This flow in a quantitative manner is represented by the heat flux vector $q$.

The total thermal resistance of the considered bed $R_{t o}$ is calculated as a parallel connection of the resistance of the individual sections

$$
R_{t o}=\left(2 R_{I}^{-1}+R_{I I}^{-1}\right)^{-1} .
$$

The geometric quantities to be used when defining the elementary cell are: the diameter of the bar $d_{p}$ and the width of the gap between the bars $l_{s z}$. On the basis of these two parameters, the cell height $\delta_{K}$ and the widths of side sections $l_{p}$ are designated. The values of these parameters are described by the following equations:

$$
\begin{aligned}
& \delta_{K}=\sqrt{d_{p}^{2}-\left(0.5\left(d_{p}+l_{s z}\right)\right)^{2}}, \\
& l_{p}=0.25\left(d_{p}-l_{s z}\right) .
\end{aligned}
$$

Effective thermal conductivity of the charge is defined by the equation describing conduction thermal resistance of flat layer [10]:

$$
k_{e f}=\frac{\delta_{K}}{R_{t o}} .
$$

Radiation resistance $R_{r d}$ for considered system is described by the equation [11]:

$$
R_{r d}=\frac{X_{r d}}{4 \sigma T_{m}^{3}}
$$

where $X_{r d}$ is a dimensionless coefficient with the value depending on the bar emissivity $\varepsilon_{b r}$ and the shape, as well as the relative position of the surfaces that closed the space of radiation heat transfer, $\sigma$ is the StefanBoltzmann constant and $T_{m}$ is mean absolute temperature of all surfaces.

In order to determine the value of the coefficient $X_{r d}$, the surfaces of bars $A_{1}, A_{I}$, and $A_{I I I}\left(A_{I}=A_{I I I}\right)$ and the apparent surface $A_{s z}$ (which concerns the gap) are taken into account. Because surface $A_{s z}$ does not reflect radiation, its emissivity is assumed to be equal to 1 . 
Due to the assumption about the temperature field, this system boils down to the system of two surfaces, the upper surface $A_{1}$ and the lower surface $A_{2}$, where

$$
A_{s z}=A_{I}+A_{s z}+A_{I I I} \text {. }
$$

Surface $A_{2}$ is assigned the equivalent emissivity $\varepsilon_{e q}$, which is determined as a weighted average

$$
\varepsilon_{e q}=\frac{A_{I}}{A_{2}} \varepsilon_{b r}+\frac{A_{s z}}{A_{2}} \varepsilon_{s z}+\frac{A_{I I I}}{A_{2}} \varepsilon_{b r} .
$$

Thanks to such approach, the radiative heat transfer in the considered cell is reduced to heat exchange between two surfaces closing the space, one of which is convex and the other concave. For such system effective emissivity and coefficient are represented by the following expressions:

$$
\begin{aligned}
\varepsilon_{e f} & =\left[\frac{1}{\varepsilon_{b r}}+\frac{A_{1}}{A_{2}}\left(\frac{1}{\varepsilon_{e q}}-1\right)\right]^{-1}, \\
X_{r d} & =\frac{2 l_{p}+l_{s z}}{A_{1} \varepsilon_{e f}} .
\end{aligned}
$$

Complete mathematical description of all thermal resistances occurring in the discussed model of effective thermal conductivity is presented in the monograph [12].

In order to show the share of radiation in a heated bar bundle, calculations of the coefficient $k_{e f}$ for two variants were made. In the first variant, the model takes into account all heat transfer mechanisms. In the second variant, thermal radiation has been omitted, taking into account only heat conduction in bars and gas and contact conduction - coefficient calculated in this way to distinguish was marked as $k_{s u}$.

The percentage share of thermal radiation $Q_{r d}$ for a given case was calculated from the relation

$$
Q_{r d}=\frac{k_{e f}-k_{s u}}{k_{e f}} \times 100 \% .
$$

The difference between the $k_{e f}$ and $k_{s u}$ coefficients is the so-called radiation thermal resistance $k_{r d}$. This parameter is commonly used to analysis of thermal radiation in porous media $[6,7,13]$.

In the calculation two bar diameters (10 and $30 \mathrm{~mm})$, three emissivity values $(0.5,0.7$, and 0.9$)$ and two porosities were taken into account. The porosity of the bundle in the adopted geometric model is a function of the gap width $l_{s z}$ - two values of this parameter were assumed: $0.1 d_{p}$ and $0.4 d_{p}$. These values of $l_{s z}$ correspond to the porosities 0.145 and 0.214 , respectively. All calculations were performed for the temperature range of $200 \div 700^{\circ} \mathrm{C}$. The model also takes into account that the bar thermal conductivity, the gas thermal conductivity, and the thermal contact resistance change with temperature.

\section{Results and discussion}

Analyzing the results of calculations, as first the $k_{e f}$ coefficient values were presented (Figs. 4 and 5). Figure 4 applies to a $10 \mathrm{~mm}$ bar bundle, while Fig. 5 refers to $30 \mathrm{~mm}$ bar bundle.
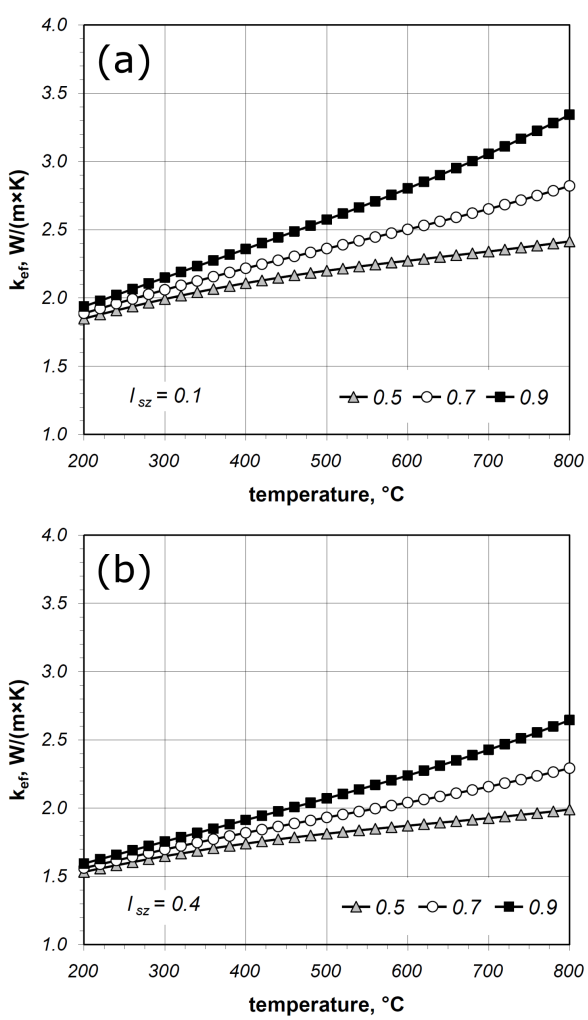

Fig. 4. Effective thermal conductivity of $10 \mathrm{~mm}$ bar bundle: (a) results for $l_{s z}=0.1 d_{p}$, (b) results for $l_{s z}=0.4 d_{p}$.
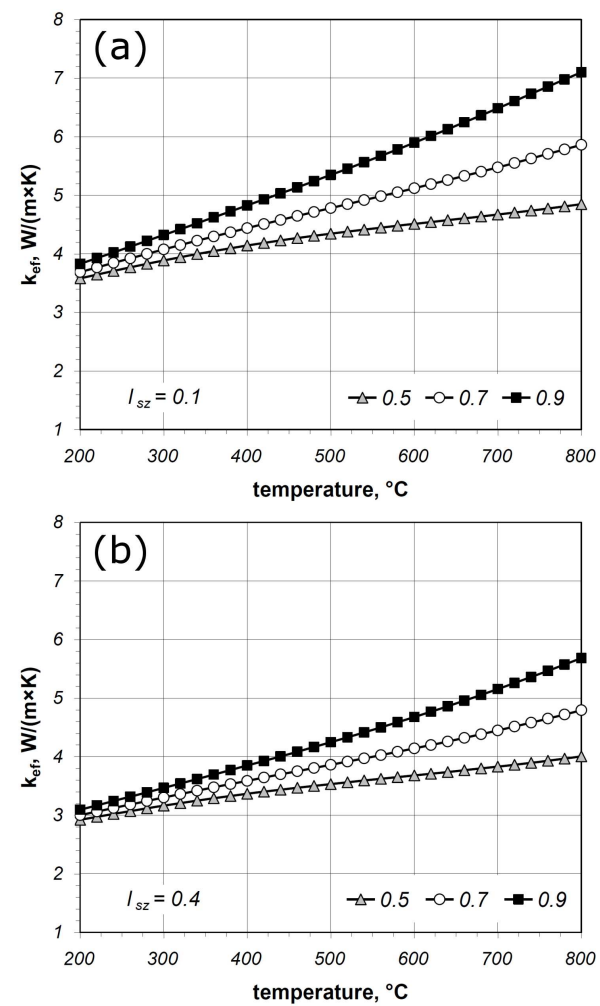

Fig. 5. Effective thermal conductivity of $30 \mathrm{~mm}$ bar bundle: (a) results for $l_{s z}=0.1 d_{p}$, (b) results for $l_{s z}=0.4 d_{p}$. 
TABLE I

The ranges of $k_{e f}$ value of 10 and $30 \mathrm{~mm}$ bar bundles.

\begin{tabular}{c|c|c}
\hline \hline$d_{p}[\mathrm{~mm}]$ & $l_{s z}$ & $k_{e f}[\mathrm{~W} /(\mathrm{m} \mathrm{K})]$ \\
\hline 10 & $0.1 d_{p}$ & $1.88 \div 3.34$ \\
10 & $0.4 d_{p}$ & $1.53 \div 2.64$ \\
30 & $0.1 d_{p}$ & $3.58 \div 7.01$ \\
30 & $0.4 d_{p}$ & $2.92 \div 5.68$
\end{tabular}

For all cases, the effective thermal conductivity increases as a function of temperature. At the same time, the dynamics of this increase is the emissivity function. This feature indicates a significant influence of thermal radiation on the total heat flow in the bundle. On the other hand, the increase of bundle porosity for both bar diameters causes the value of $k_{e f}$ to decrease. In percentage terms this decrease, depending on the emissivity value, ranges from $20 \%$ to $25 \%$. The ranges of values of $k_{e f}$ for this case are summarized in Table I.

From this statement it can be seen that the parameter that most strongly affects the effective thermal conductivity value is the bar diameter.

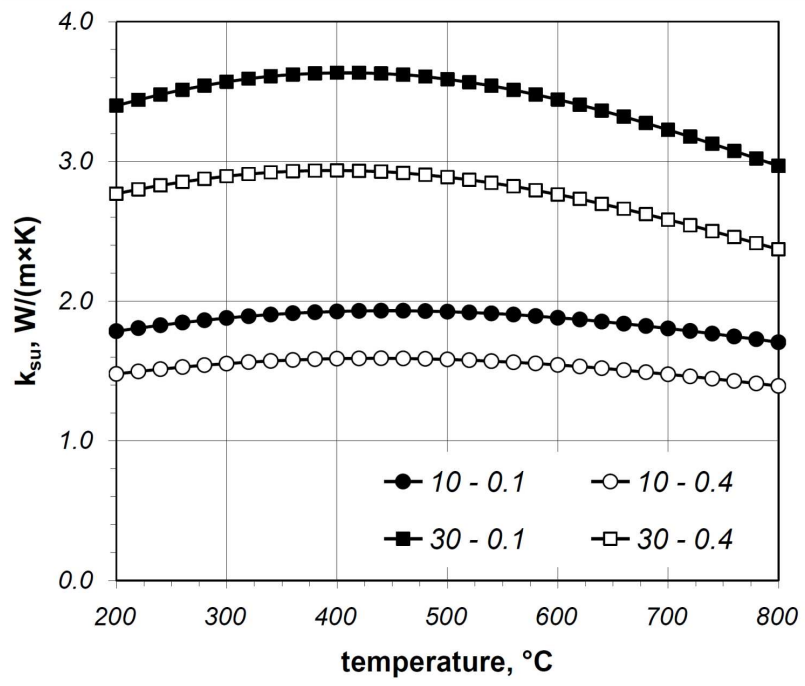

Fig. 6. Substantial thermal conductivity $k_{s u}$ of the considered bar bundles.

TABLE II

The ranges of $k_{s u}$ value of 10 and $30 \mathrm{~mm}$ bar bundles.

\begin{tabular}{c|c|c}
\hline \hline$d_{p}[\mathrm{~mm}]$ & $l_{s z}$ & $k_{s u}[\mathrm{~W} /(\mathrm{m} \mathrm{K})]$ \\
\hline 10 & $0.1 d_{p}$ & $1.71 \div 1.93$ \\
10 & $0.4 d_{p}$ & $1.39 \div 1.59$ \\
30 & $0.1 d_{p}$ & $2.97 \div 3.63$ \\
30 & $0.4 d_{p}$ & $2.37 \div 2.93$
\end{tabular}

Results of calculations for the second variant, regarding to substantial thermal conductivity $k_{s u}$ are shown in Fig. 6. Because in this situation the emissivity as variable is eliminated, the results of all cases are presented in one graph. Results denotation in Fig. 6 is - for example: 10-0.1 applies to a bundle of $10 \mathrm{~mm}$ bars with a gap width of $0.1 d_{p}$. The value of $k_{s u}$ increases as a function of the bar diameter and decreases as a function of bundle porosity. This coefficient increases also with temperature, but only up to around $400^{\circ} \mathrm{C}$. After exceeding this temperature, it begins to decrease. At the same time, the $k_{s u}$ values at $200^{\circ} \mathrm{C}$ for all cases are slightly higher than the values obtained at $800^{\circ} \mathrm{C}$. The ranges of values of $k_{s u}$ are summarized in Table II.

Figures 7 and 8 shows the values of radiative thermal conductivity $k_{r d}$. As can be seen, the value of this coefficient strongly depends on the temperature. It also grows as a function of emissivity and bar diameter, while decreases with increase of bundle porosity. The maximal values of $k_{r d}$ coefficient are summarized in Table III.
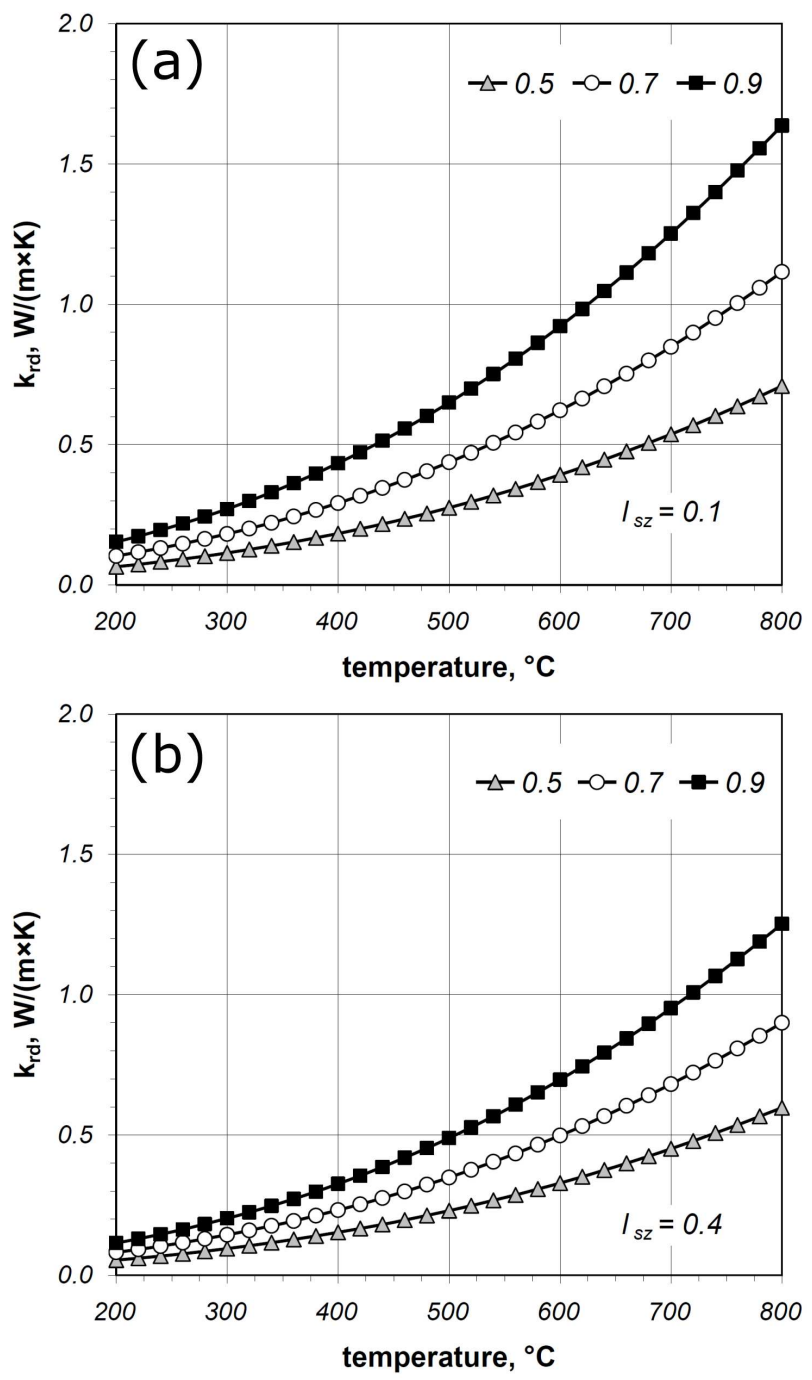

Fig. 7. Radiation thermal conductivity $k_{r d}$ of $10 \mathrm{~mm}$ bar bundle: (a) results for $l_{s z}=0.1 d_{p}$, (b) results for $l_{s z}=0.4 d_{p}$. 

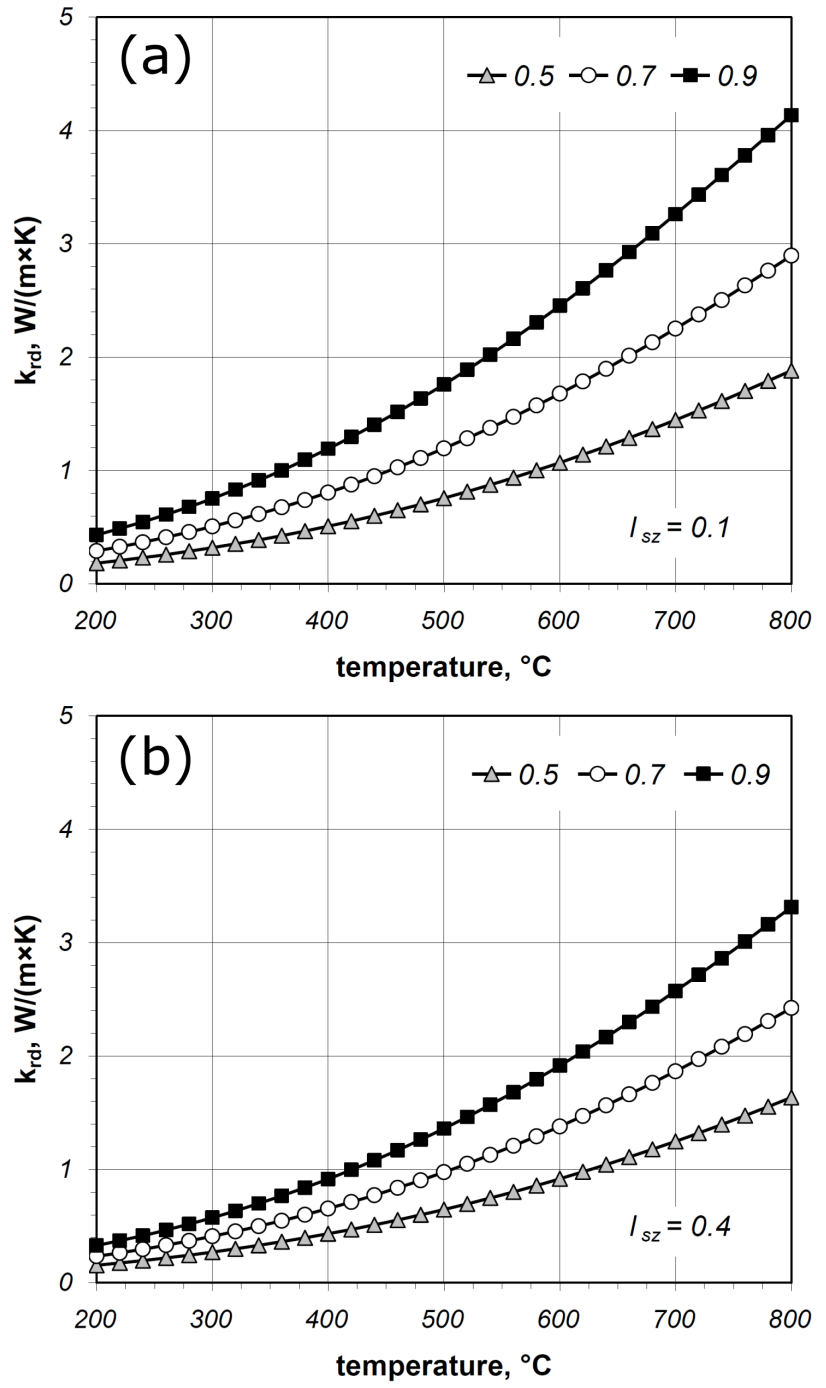

Fig. 8. Radiation thermal conductivity $k_{r d}$ of $30 \mathrm{~mm}$ bar bundle: (a) results for $l_{s z}=0.1 d_{p}$, (b) results for $l_{s z}=0.4 d_{p}$.

TABLE III

The maximal values of $k_{r d}$ coefficient.

\begin{tabular}{c|c|c}
\hline \hline$d_{p}[\mathrm{~mm}]$ & $l_{s z}$ & $k_{r d}[\mathrm{~W} /(\mathrm{m} \mathrm{K})]$ \\
\hline 10 & $0.1 d_{p}$ & 1.63 \\
10 & $0.4 d_{p}$ & 1.25 \\
30 & $0.1 d_{p}$ & 4.13 \\
30 & $0.4 d_{p}$ & 3.31
\end{tabular}

Figures 9 and 10 illustrate the values of thermal radiation share $Q_{r d}$ calculated using Eq. (8). The values for $10 \mathrm{~mm}$ bar bundle in considered temperature range, depending on the gap width $l_{s z}$ and bar emissivity $\varepsilon$ are summarized in Table IV, whereas Table V shows the values of $Q_{r d}$ averaged for the whole temperature range.

The values of $Q_{r d}$ parameter obtained for the bundle of $30 \mathrm{~mm}$ bars are shown in Tables VI and VII.
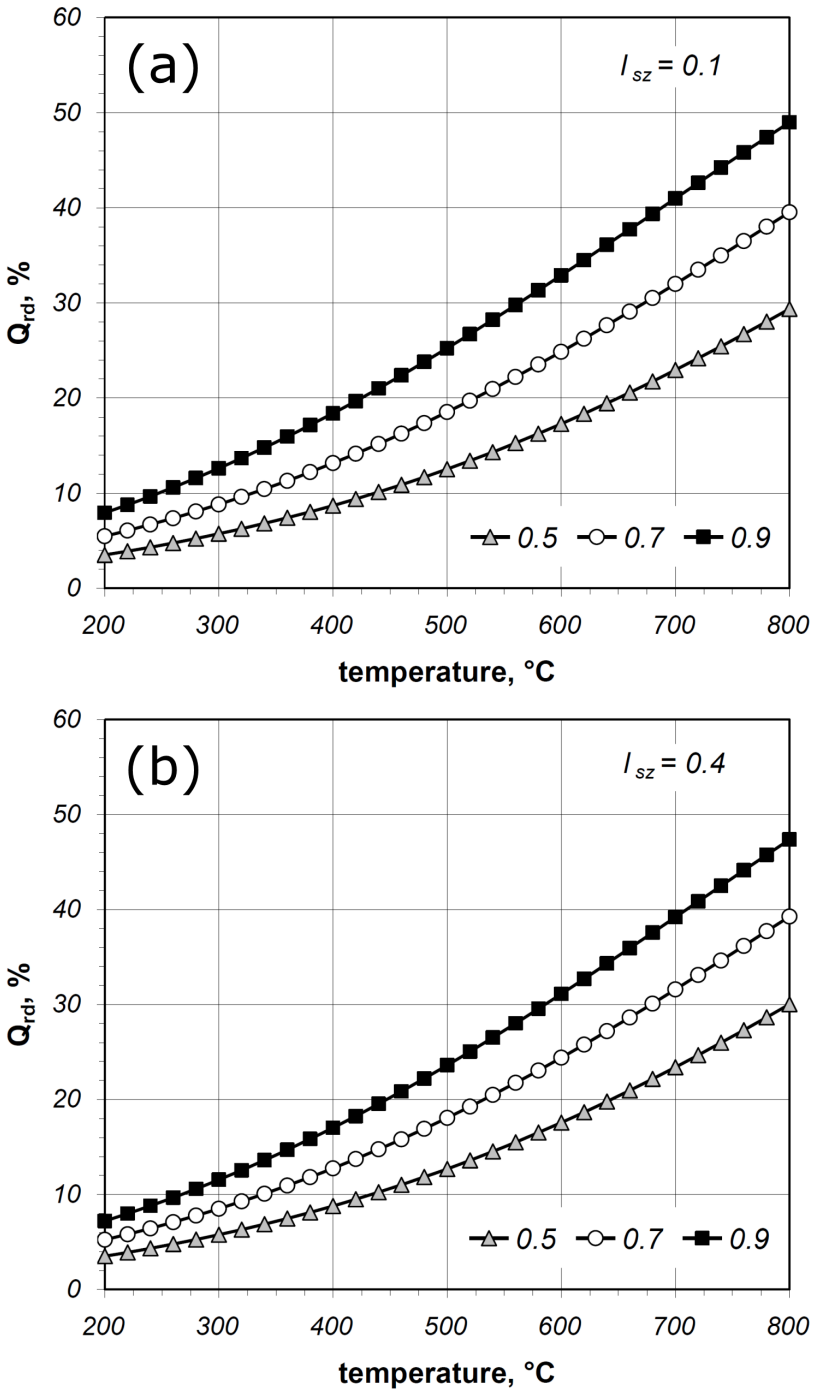

Fig. 9. Percentage share of radiation in the $10 \mathrm{~mm}$ bar bundle: (a) results for $l_{s z}=0.1 d_{p}$, (b) results for $l_{s z}=0.4 d_{p}$.

TABLE IV

The ranges of $Q_{r d}$ for $10 \mathrm{~mm}$ bar bundle.

\begin{tabular}{c|c|c}
\hline \hline$l_{s z}$ & $\varepsilon$ & $Q_{r d}[\%]$ \\
\hline $0.1 d_{p}$ & 0.5 & $3.5 \div 30.0$ \\
$0.1 d_{p}$ & 0.9 & $7.2 \div 47.4$ \\
$0.4 d_{p}$ & 0.5 & $3.5 \div 29.4$ \\
$0.4 d_{p}$ & 0.9 & $7.9 \div 49.0$
\end{tabular}

TABLE V

The values of $Q_{r d}$ for $10 \mathrm{~mm}$ bar bundle averaged for the whole temperature range.

\begin{tabular}{c|c|c}
\hline \hline$l_{s z}$ & $\varepsilon$ & $Q_{r d}[\%]$ \\
\hline $0.1 d_{p}$ & 0.5 & 14.0 \\
$0.1 d_{p}$ & 0.9 & 26.4 \\
$0.4 d_{p}$ & 0.5 & 14.2 \\
$0.4 d_{p}$ & 0.9 & 25.0
\end{tabular}



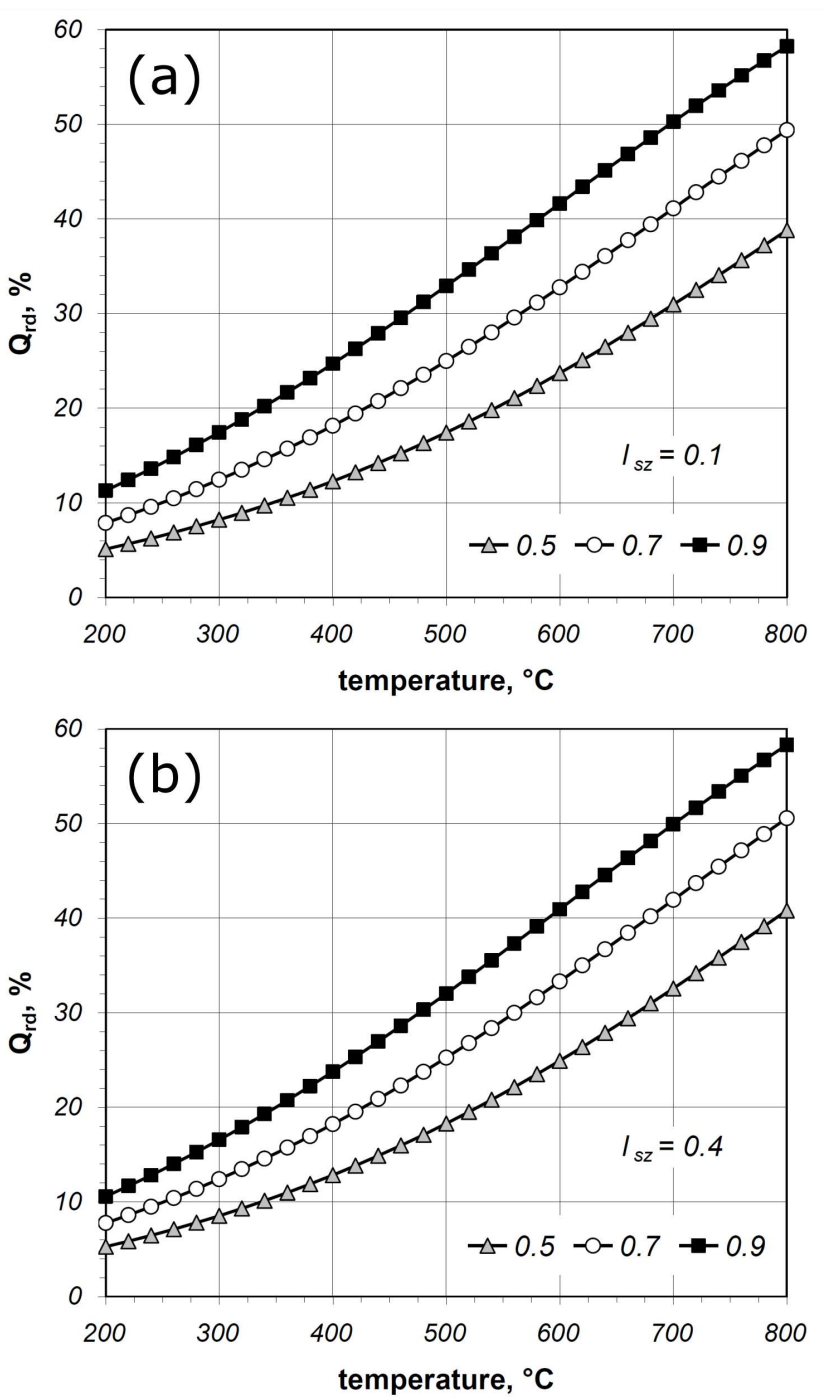

Fig. 10. Percentage share of radiation in the $30 \mathrm{~mm}$ bar bundle: (a) results for $l_{s z}=0.1 d_{p}$, (b) results for $l_{s z}=0.4 d_{p}$.

TABLE VI

The ranges of $Q_{r d}$ for $30 \mathrm{~mm}$ bar bundle.

\begin{tabular}{c|c|c}
\hline \hline$l_{s z}$ & $\varepsilon$ & $Q_{r d}[\%]$ \\
\hline $0.1 d_{p}$ & 0.5 & $5.1 \div 38.8$ \\
$0.1 d_{p}$ & 0.9 & $11.3 \div 58.2$ \\
$0.4 d_{p}$ & 0.5 & $5.3 \div 40.8$ \\
$0.4 d_{p}$ & 0.9 & $10.6 \div 58.3$
\end{tabular}

The obtained results show that the share of thermal radiation varies within very wide limits and depends on a number of parameters. The factor that has the strongest influence on the $Q_{r d}$ value is temperature. Up to $200^{\circ} \mathrm{C}$ the value of $Q_{r d}$ does not exceed $10 \%$. While in $800^{\circ} \mathrm{C}$, the value of $Q_{r d}$ can exceed even $50 \%$.

The bar emissivity is another important parameter. This quantity expresses the ability of the surface to radiation emission, and in the considered case it depends
TABLE VII

The values of $Q_{r d}$ for $30 \mathrm{~mm}$ bar bundle averaged for the whole temperature range.

\begin{tabular}{c|c|c}
\hline \hline$l_{s z}$ & $\varepsilon$ & $Q_{r d}[\%]$ \\
\hline $0.1 d_{p}$ & 0.5 & 19.1 \\
$0.1 d_{p}$ & 0.9 & 33.6 \\
$0.4 d_{p}$ & 0.5 & 20.1 \\
$0.4 d_{p}$ & 0.9 & 32.9
\end{tabular}

on the physical condition of the bars surface being processed. The emissivity is the higher, the stronger the steel surface is corroded or oxidized. Emissivity of slightly oxidized steel surfaces is about 0.7 [14].

The third parameter that contributes to the increase in the $Q_{r d}$ value is the bar diameter. In a bundle of $10 \mathrm{~mm}$ bars, the average value of $Q_{r d}$ is from $14 \%$ to $26 \%$, whereas for a bundle of $30 \mathrm{~mm}$ bars this range is from $19 \%$ to $33 \%$. The value of the $Q_{r d}$ in very small degree depends on the porosity. A change in the value of this parameter from 0.145 to 0.214 - which gives an increase by more than $47 \%$ - results in a $Q_{r d}$ change of only about $1 \%$.

\section{Summary and conclusions}

Results of make calculations showed that the share of thermal radiation in the heated round bar bundle varies within wide limits. The value of $Q_{r d}$ increases in the function of: temperature, emissivity, and bars diameter, while the effect of porosity on the $Q_{r d}$ value is negligible.

The obtained results shows also that at analysis of heat flow in the heat-treated bar bundles, thermal radiation phenomena cannot be ignored. In the heat treated bundle of round bars by this mechanism of heat transfer a significant amount of thermal energy is transferred.

\section{References}

[1] S.S. Sahay, K. Krishman, Iron Steelmak. 34, 89 (2007).

[2] D. Musiał, Arch. Metall. Mater. 1, 63 (2013).

[3] T.R. Rao, G.J. Barth, J.R. Miller, Iron Steel Eng. 60, $22(1983)$.

[4] Y. Zuo, W. Wu, X. Zhang, L. Lin, S. Xiang, T. Liu, L. Niu, X. Huang, Heat Transf. Asian Res. 30, 615 (2001).

[5] S.S. Sahay, P.C. Kapur, Iron Steelmak. 34, 262 (2007).

[6] M. Kaviany, Principles of Heat Transfer in Porous Media, 2nd ed., Springer-Verlag, New York 1995.

[7] W. Van Antwerpen, C.G. du Toit, P.G. Rousseau, Nucl. Eng. Des. 240, 1803 (2010).

[8] X. Zhang, F. Yu, W. Wu, Y. Zuo, Int. J. Thermophys. 24, 1395 (2003).

[9] R. Wyczolkowski, Proced. Eng. 157, 185 (2016).

[10] Y.A. Cengel, Heat and Mass Transfer - A Practical Approach, 3rd ed., McGraw-Hill, New York 2007. 
[11] R. Wyczolkowski, D. Urbaniak, J. Thermophys. Heat Transf. 30, 721 (2016).

[12] R. Wyczolkowski, Modelling of effective thermal conductivity of the steel porous charge using the thermal resistance concept, Series Monographs, Vol. 67, Czestochowa University of Technology, Czestochowa 2017 (in Polish).
[13] G. Breitbach, H. Barthels, Nucl. Technol. 49, 392 (1980).

[14] F.M. Modest, Radiative Heat Transfer, 3rd ed., Academic Press, Amsterdam 2013. 